\title{
Design of a Neural Controller Applied a Level System in Hart Protocol
}

\author{
MURILLO FERREIRA DOS SANTOS ${ }^{1}$, KAMILA PERES ROCHA ${ }^{2}$, MARLON JOSÉ DO \\ CARMO $^{3}$ \\ ${ }^{1}$ Intelligent Robotic Group - GRIn, Juiz de Fora Federal University, Juiz de Fora, Minas Gerais - Brazil \\ ${ }^{2}$ CEFET-MG Campus III Leopoldina, Department of Electronics, Leopoldina, Minas Gerais - Brazil \\ E-mail: ${ }^{1}$ murilloferreiradossantos@gmail.com, ${ }^{2}$ marloncarmo@ieee.org
}

\begin{abstract}
This paper presents a design of a neural controller for industrial level systems. The level process has an asymmetric dynamic and its control is not a simple process of performing. This work presents an advanced control technique using intelligent control with artificial neural networks. The proposal is to implement a network of multilayer perceptron with a PI controller for controlling a level system based on a SMAR ${ }^{\circledR}$ didactic plant with Hart protocol. The control strategy is implemented with Matlab ${ }$. This software makes a communication with the plant through OPC (OLE for process control). The project demonstrates the practical feasibility and applicability of intelligent tools industrial systems, thus generating a gain in experimental learning, commonly found in the labor market.
\end{abstract}

Keywords: Hart Networks, Artificial Neural Networks, Process Control, Nonlinear systems, Industrial Networks.

\section{INTRODUCTION}

Nowadays, industries need more analysis and control of their processes in order to get better quality and speed, lower cost, and flaws. To assist in the design and analysis of the functioning of control systems, it is necessary to obtain their identification to apply a good control strategy to reduce uncertainty and improve the performance of a system.

With the development of technology, one of the most widespread control and process automation is the Artificial Intelligence. Modeling of intelligent control look for to reduce uncertainty and to improve the performance of a closed-loop system [1].

There are some techniques of artificial intelligence that can be inserted into the grid engineering to control processes such as neural networks, fuzzy logic, neural-fuzzy.

Artificial neural networks are computational models inspired in the nervous system of living beings. It have the capability of acquiring and maintaining knowledge (based on information), and can be defined as a set of processing units, characterizeed by artificial neurons which are interconnected by a large number of interconnections (artificial synapses), the same being represented by vectors / matrices of synaptic weights [2].

Neural networks can be used to solve various problems related to engineering and science. The applicability of this technique is very broad and among them, there is a use for the control systems to the quality, safety and efficiency.

More specifically, the most attractive feature of artificial neural networks is the capability of use it in many problems, like high skills in mapping nonlinear systems and learning the behaviors involved from the information (measurements, samples or patterns).

To design the controller of a system level, it was used the SMAR ${ }^{\circledR}$ didactic plant with Foundation Fieldbus protocol. A didactic plant simulates processes commonly found in industry. It was used the software MATLAB ${ }^{\circledR}$ that performs the communication with the computer via OPC Protocol (OLE for Process Control). Through this communication, all commands given directly by the 
software eliminating the use of PLC (Programmable Logic Controller), making it as

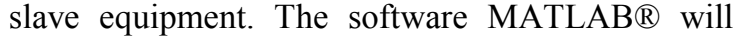
monitor and write values directly in the devices of the plant.

The objective of this paper is to get the design of a neural PI controller for industrial level systems. Is used artificial neural networks to control a level system, which simulates industrial processes in smaller scale.

This paper is divided as follows: The Section II describes the characteristics of the level system adopted; The Section III says about the two protocol of communication; The Section IV explains how this paper was developed, taking about the procedure of the experiments; The Section V shows some results produced; The Section VI presents the conclusion of the paper.

\section{$2 \quad$ SMAR $^{\circledR}$ DIDACTIC PLANT}

The purpose of the SMAR $\AA$ didactic plant (shown in Figure 1) is demonstrate didactically the implementation of control loops commonly found in industries.

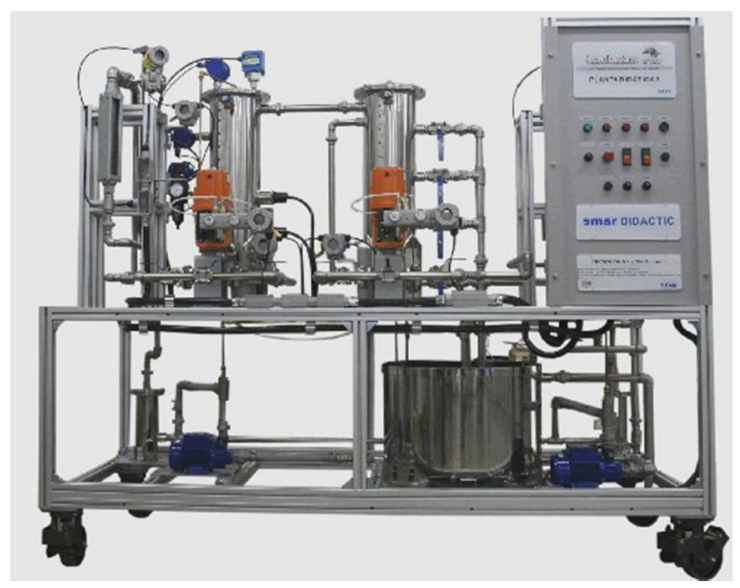

Fig. 1. SMAR $®$ Didactic Plant operated by HART Protocol.

The technology used for this demonstration is a plant with Foundation Fieldbus protocol. It consists of a workstation by PC type, which is connected to the didactic plant via TCP/IP. The Foundation Fieldbus Bridge does the interface between the Ethernet and field bus. This bus is connected to all continuous instruments, which are the pneumatic valve positioners, level, flow and temperature transmitters [3].

The level control is one of the most frequently found in the industry. On SMAR $\AA$ plant, the level measurement is fair by differential transmitter (model LD 302), which is based on principles of hydrostatics [4].

The law of Conservation of Mass is used when it is operated in open loop to keep the tank level stabilized, which for a constant flow is necessary that the outflow is increased to match the input flow. In closed loop, the input flow is controlled by the control valve and the outlet flow is changed by a manual valve [3]. This process is illustrated below in Figure 2, which shows a block diagram of a control level loop. In Figure 3 is presented a schematic control level loop with some equipment:

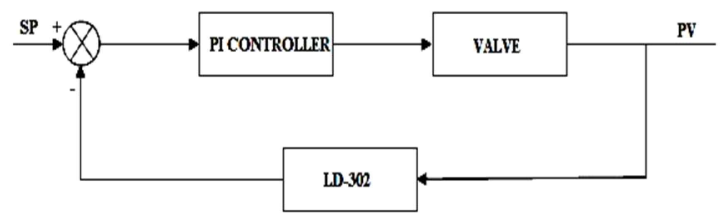

Fig. 2. Block diagram of a control level loop.

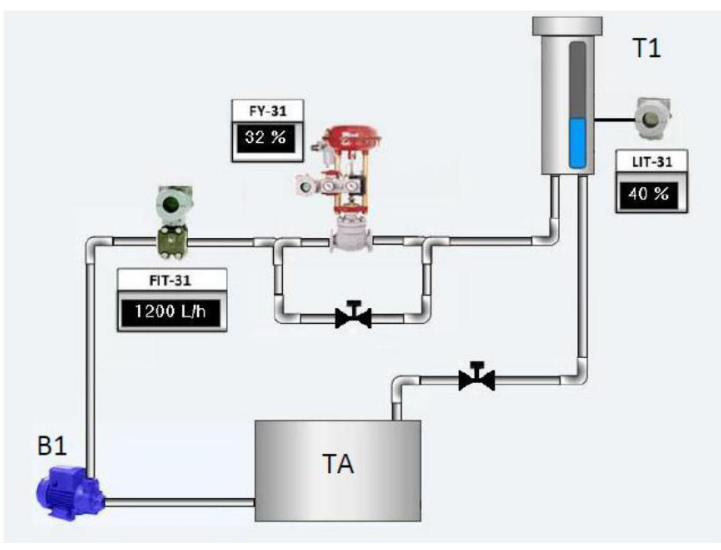

Fig. 3. Schematic control level.

The pump B1 to the tank T1 pumps the water from the tank TA. The water passes through the control valve FY-31, which the flow is measured through the flow meter FIT-31.

The opening in the bottom of the tank simulates the water consumption and is made up through a manifold valve.

\section{HART AND OPC PROTOCOL}

The evolution of electronic sensors made those reach the category of microprocessor smart sensors, contributing to the insertion of the first digital signal in field instruments, HART (Highway Addressable Remote Transducer) [5].

The HART protocol is currently one of the most used protocols in level industries, such as interconnections of equipment in smart fields. It was created by Rosemount in the United States in 
the middle of 1980 s as a proprietary protocol, i.e., it was closed, where he later became an open standard protocol and has evolved since then [6].

Both the 4-20 $\mathrm{mA}$ analog and HART digital can be carried on the same bus. The 4-20 mA standard protocol was developed in 1972 in an attempt to standardize the industrial networks, which despite being old when compared to other standards, but they are still widely used [7].

A few decades ago, there was a big problem in the consistency protocol at the application layer for equipment and plant floor systems from different manufacturers and technologies [8].

From the fusion of several technologies, it was created the OPC protocol (OLE for Process Control) to solve the problem of the multiplicity of existing drivers and only catered to specific versions. From the OPC, a manufacturer of controllers and field instruments of all technologies always provide your equipment with an OPC server [9].

Those applications need only know how to look for data from the OPC server, bypassing the implementation of the device where the server needs to provide data in a single format, which actually makes the task of communication so much easier [8].

\section{PROCEDURE OF THE EXPERIMENT}

It can be observed that the process has a nonlinear asymmetrical dynamic, in other words, the system response (made by a unit step input) has rapid growth and slow early region near steady state. Thus, a neural PI (Proportional and Integral) controller is designed to act on the water valve plant, in order to maintain the predetermined level.

Since the system has high complexity and its structure is unknown, its analysis has limited relationships between input and output values. In theory systems, they are called of black box and the most common learning algorithm is neural network, often developed in backpropagation version. For this project, it was used the Levenberg-Marquardt method. It is based on the delta rule, where the adjustments of weights are made using the gradient method. The activation function of the logistics network was chosen because of its features [10].

A neural network is trained to classify the plant behavior, where the synaptic weights are adjusted according to the data presented.

The next step is to select a neural network model. One of the most used families to define nonlinear models are perceptrons multi-layers.

It is important to specify the number of hidden layers and the number of neurons in the network.
With two hidden layers is possible to approximate any mathematical function and further classifying patterns which are in any kind of geometric regions [11].

The controller will be included in the proportional and integral control, so two neural networks were implemented. One network was used to represent the proportional error between a reference and the results and another to respond to the integral of the error [12].

A pattern vector is generated and it is assumed to represent the error between a reference and the actual output of the plant where an answer is obtained damped sine, which is typical of the behavior of plants where the controller will be implemented [9].

The software MATLAB ${ }^{\circledR}$ was used for the implementation and training of network as well as direct control via OPC toolbox. The Figure 4 shows the block diagram of the experiment operation.

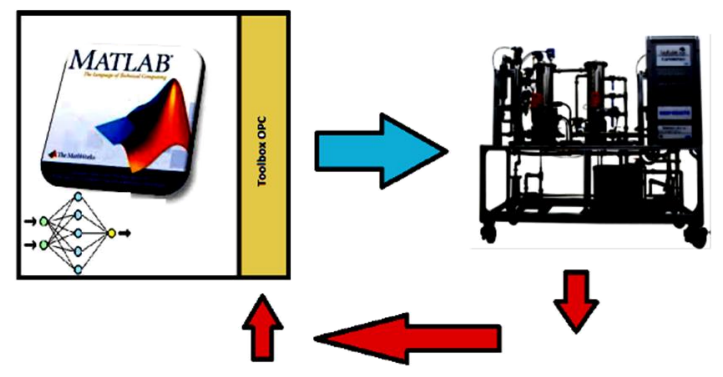

Fig. 4. Communication system - MATLAB®/Neural Controller versus Didactic Plant.

In this network training as a controller, it was used a damped sinusoid as vector training for proportional gain and the gradient of this training to obtain the integral action as presented below in Figure 5:

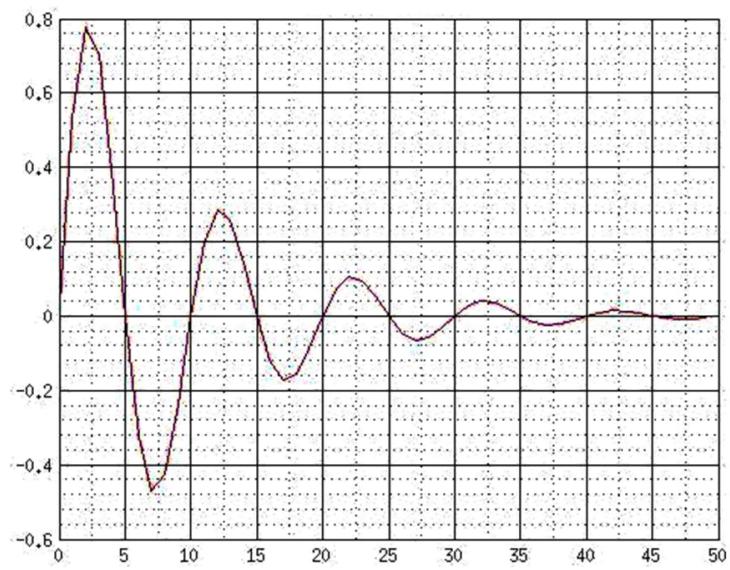

Fig. 5. Training signal of a sinusoid for the neural network. 
As it can be seen in Fig. 5, the oscillations are gradually damped in each cycle, which in fact leads to neural network to generate synaptic weights geared to perform the same aspect of response when exposed to situations in the real operating situations.

Then, two types of networks feed forward are created [12]. The two networks were configured with ten neurons in the hidden layer and one neuron in the output one. The values for the network input is in percentage, taking care the actuator in control (valve of water opening the plant you want to get control of tank level). As learning function, it was used backpropagation, as performance factor, it was chosen the mean squared error. The Figure 6 shows

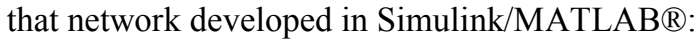

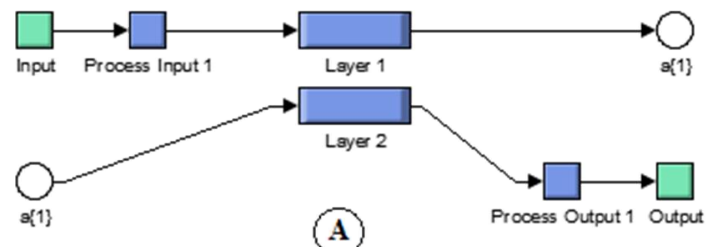

(A)
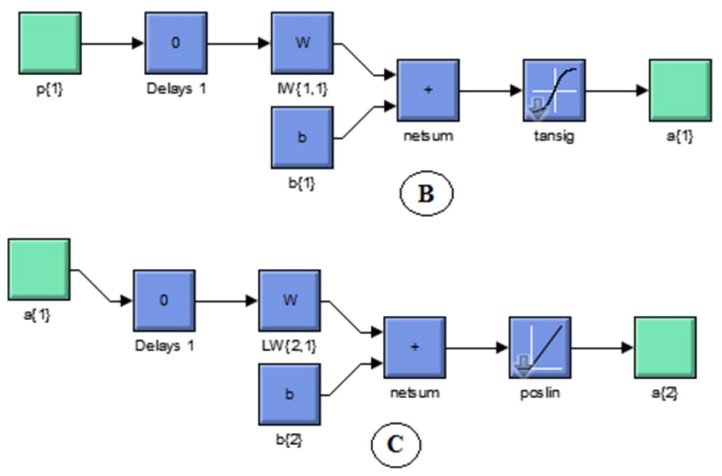

Fig. 6. A) Control loop made up in Simulink; B) Layer 1; C) Layer 2.

The networks are initialized to be trained later. It's set the training period for a certain amount of epochs and the value of the mean squared error desired.

After training, simulations are made up to verify that the networks learned the system behavior.

Tests are performed on the networks in order to observe their performance as controllers. Thus, one should choose an arbitrary training function to simulate the network capacity for integrated and proportional control [12].

The last part is to realize some trials for variations in the valve opening to plot the results in proportional and integral gains. Both these gains are variable, and the scaling can be performed in a manner which provides the desired response.

However, some aspects of the control loop were inserted for some comparisons can be done. Among them, the most significant technique is the use of anti reset windup, which is meant to mitigate the effect of the integral action when saturation in the physical system occurs. To describe it, a block diagram is presented in Figure 7 showing this topology:

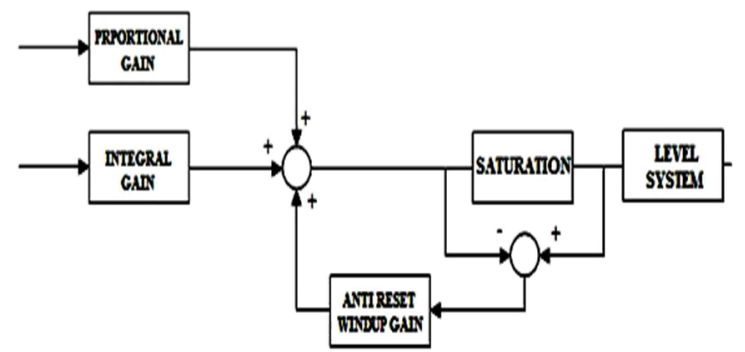

Fig. 7. Block diagram which represents the anti reset windup technique.

\section{RESULTS}

Based on robustness and versatility, this method (neural PI controller) is meant to facilitate the understanding of various aspects.

For the experiments presented in this paper, the system, which is being controlled, has dynamic similar of the presented below in Figure 8:

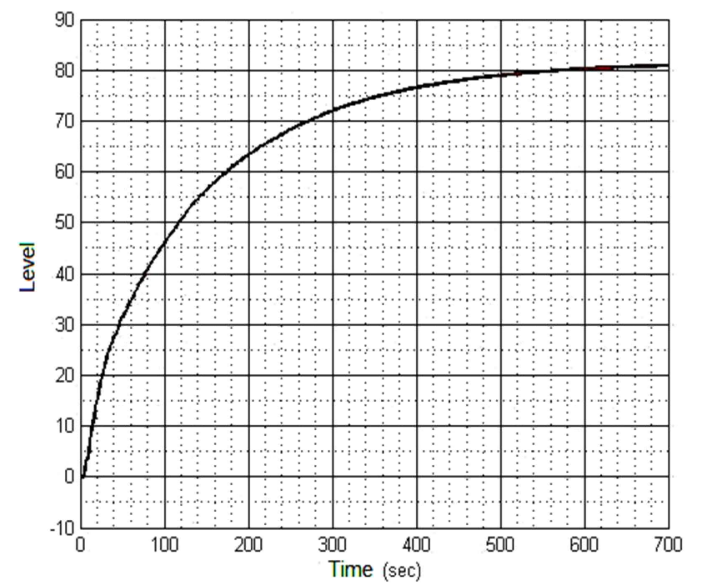

Fig. 8. Dynamic of the level system to be controlled with a specific opennig of the control valve.

For this test made in Fig. 8, the manifold valve was set for one specific flow. If it is changed, this dynamic response changes as well.

To compare the responses, a traditional technique was already implemented at the same didactic system, for example, the Haalman PI controller. For the same aspect of response, it's shown below in Fig. 9 the results of this controller adopting SP (Set Point) of $40 \%$ [13]. 


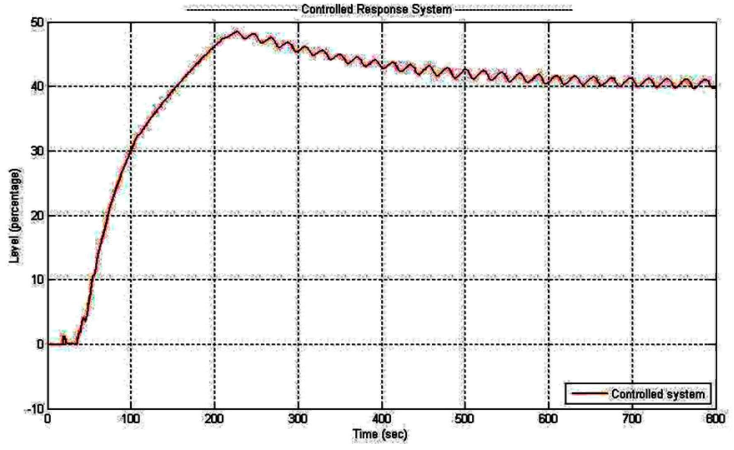

Fig. 9. Dynamic of the level system to be controlled with a specific opennig of the control valve.

Then, taking the same characteristics as taken in Fig. 9, three different situations of gains and SP were adopted, resulting the Figures 10, 11 and 12, according to Table 1 shown below:

Table: 1 Simulation data per Figure

\begin{tabular}{|c|l|l|l|}
\hline \multirow{2}{*}{$\begin{array}{c}\text { Fi } \\
\text { gure }\end{array}$} & \multicolumn{3}{|c|}{ Simulation Data } \\
\cline { 2 - 4 } & $\begin{array}{c}\text { Proporti } \\
\text { onal Gain }\end{array}$ & $\begin{array}{c}\text { Integral } \\
\text { Gain }\end{array}$ & $\begin{array}{c}\text { Set } \\
\text { Point (SP) }\end{array}$ \\
\hline 9 & 5 & 1 & 50 \\
\hline 10 & 10 & 1 & 27 \\
\hline 11 & 10 & 1 & 50 \\
\hline
\end{tabular}
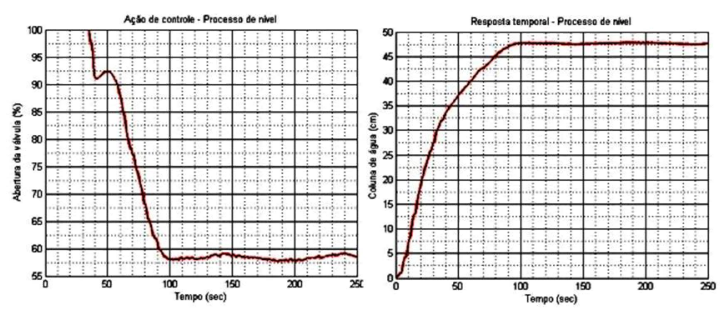

Fig. 10. Control action (Left); Response time (Right).
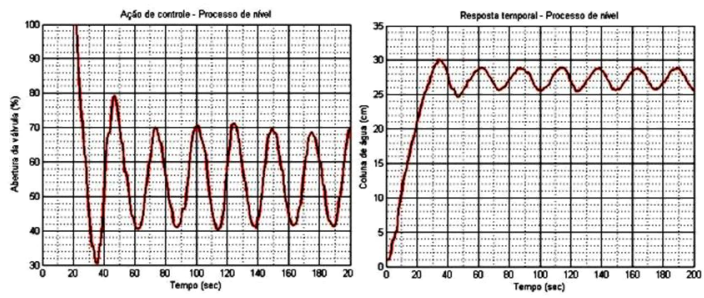

Fig. 11. Control action (Left); Response time (Right).
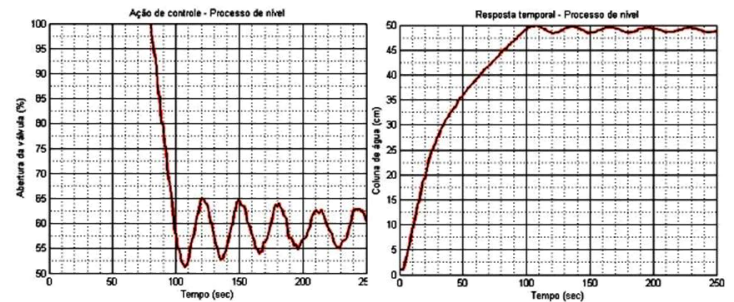

Fig. 12. Control action (Left); Response time (Right).
As it can be understood in Figure 10, the system displays the performance of the temporal response of the neural PI controller, which has no one overshoot for the parameters inserted.

For Figure 10, the system shows oscillations through the set point, which leads us to conclude that the gains should be adjusted to improve the results.

In Figure 11, using the same gains of Fig. 10, just changing the set point of the system, it can be seen that the oscillations are virtually nil, within acceptable.

The control action printed of the system (in Figure 11) showed more aggressive if compared to other ones, this is due to the fact that larger gains and set point.

\section{CONCLUSIONS}

After the application of this technique (neural proportional and integral controller) which simulates the actual level process (so much found in industry), it can be noted that some advantages of using artificial intelligence techniques to control level systems.

Currently, these tools such as artificial neural networks represent much knowledge in the area of intelligent systems for its wide applicability in many areas. From the study and application of control real nonlinear system, it can be affirmed that efficiency and accuracy of understand the system behavior and verify the existence of options in control strategies that can be attractive alternatives if compared to conventional control loops.

The experiments of real systems using artificial neural networks show that this practice can be used in learning, assisting in the training of professionals and optimizing systems levels seeking improvements in the industrial production.

\section{ACKNOWLEDGMENT}

The authors would like to thank MEC / SESu, ENDF, CAPES, FAPEMIG, CEFETMINAS Foundation and CEFET-MG by supporting the development of this work.

\section{REFERENCES}

[1] BRUNETTE, E. S.; FLEMMER, R. C.; FLEMMER, C. L.; A review of artificial intelligence, ICARA - International Conference on Autonomous Robots and Agents, ISBN: 978-1-4244-2712-3, Wellington, New Zealand, February, 2009, pp. 385-392. 
M. F. D. Santos et al. / International Journal of Computer Networks and Communications Security, 2 (1), January 2014

[2] CUNBIN, L.; KECHENG, W., Transmission Theory of the Risk Neural Network, International Conference on Network and Parallel Computing Workshops, ISBN: 978-07695-2943-1, Liaoning, China, 2007, pp. 909914 .

[3] KARAMI, J.; SALAHSHOOR, K.; Design and Implementatio os an Instructional Foundation Fieldbus-based Pilot Plant, ICCGI International Multi-Conference on Computing in the Global Information Technology, ISBN: 0-7695-2690-X, Bucharest, Romania, August, 2006, pp. 32.

[4] SMAR, Department of de Applications in Engennier, Datasheet - Planta Didática III, In $<$ http://www.smar.com/brasil/produtos/view.as p?id=36> Acesso em: 31 juhlo 2013.

[5] GUOCHEN, A.; ZHIYONG, M.; HONGTAO, M. SINGDONG, S., Design of Intelligent Transmitter based on HART Protocol, IEEE, ISBN: 978-1-4673-5034-1, Harbin, China, 2012, pp. 1499-1502.

[6] MULLER, I.; NETTO, J. C.; PEREIRA, C. E., WirelessHART field devices, IEEE Instrumentation \& Measurement Magazine, ISSN: 1094-6969, December, 2011, v.14, pp. 20-25.

[7] SMAR Equipamentos Ind. Ltda.. Manual de operação Planta Didática SMAR® III. 2004.

[8] JISHENG, X.; JING, B.; GUOCHENG N.; TIECHENG, P., Data acquisition system for energy management based on OPC protocol, International Conference on Mechatronic Science, Electric Engineering and Computer (MEC), ISBN: 978-1-61284-719-1, Jilin, China, August, 2011, pp. 490-493 .

[9] XIAOPING, Z; XIAOXUAN, M., Design and implementation of a uniform wireless OPC DA Server, International Conference on Mechatronic Science, Electric Engineering and Computer (MEC), ISBN: 978-1-61284-719-1, Jilin, China, August, 2011, pp. 310-313 .

[10] FLAUZINO, R. A.; SILVA, I. N.; SPATTI, D. H., Redes Neurais Artificiais para Engenharia

[11] e Ciências Aplicadas, First edition, ed. São Paulo: ArtLib, 2010.

[12] LIPPMANN, R. P., Neural nets for computing, 4 IEEE, ISBN: 1520-6149, Lexington, USA, April, 1988, v1, pp. 1-6.

[13] NGUYEN, H. T.; PRASAD, N. R.; WALKER C. L.; WALKER, E. A., A FirstCourse in Fuzzy and Neural Control, ed. CHAPMAN \& HALL/CRC, 2003.

[14] SANTOS, M. F.; CARMO, M. J.; BOCK, E. G. P. and GARCIA, E. S., Controle Haalman para sistemas de nível com dinâmica assimétrica e protocolo de comunicação HART, III Congresso Científico da Semana Tecnológica - IFSP, Bragança Paulista: IFSP, 2012.

\section{AUTHOR PROFILES:}

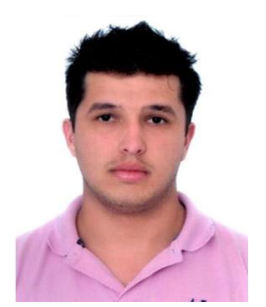

Bsc. Murillo Ferreira dos Santos received the degree in Control \& Automation engineering from CEFETMG - Brazil, in 2005. He is a student of Master degree in electrical engineering at Juiz de Fora Federal University. His interests are in industrial networks and control design.

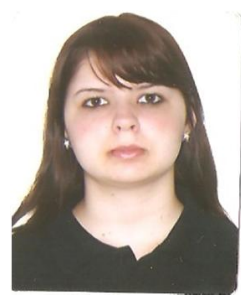

Kamila Peres Rocha is student in Control \& Automation engineering at CEFET-MG, Brazil. Her research interests include neural networks, industrial networks and System Dynamics.

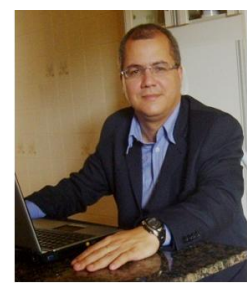

Prof. Marlon José do Carmo received the degree in Mathematics and Sciences from FIC- Brazil, in 2002, Master degree in electrical engineering in a Juiz de For a Federal University. He is a student of $\mathrm{PhD}$ degree in electrical engineering at Rio de Janeiro Federal University / COPPE. $\mathrm{He}$ is associate professor in CEFET-MG, Brazil. His interests are in industrial networks, systems identification, control design, power systems, superconductivity. 